\title{
Advances in asthma pathophysiology: stepping forward from the Maurizio Vignola experience
}

\author{
Maria R. Bonsignore ${ }^{1,2}$, Mirella Profita ${ }^{2}$, Rosalia Gagliardo², Loredana Riccobono², \\ Giuseppina Chiappara ${ }^{2}$, Elisabetta Pace ${ }^{2}$ and Mark Gjomarkaj ${ }^{2}$
}

Affiliations: ${ }^{1}$ Biomedical Dept of Internal and Specialistic Medicine (DiBiMIS), Pneumology, University of Palermo, Palermo, Italy. ${ }^{2}$ Institute of Biomedicine and Molecular Immunology (IBIM), National Research Council (CNR), Palermo, Italy.

Correspondence: Maria R. Bonsignore, DiBiMIS, University of Palermo, Via Trabucco 180, 90146 Palermo, Italy. E-mail: marisadibim.cnr.it

ABSTRACT Maurizio Vignola was a superb and innovative researcher, who wrote seminal papers on the biology of airway epithelium in asthma. Inflammation and remodelling were the main topics of his research, mostly conducted in biopsy specimens from patients with asthma of variable severity, encompassing the entire spectrum of the disease from mild to severe asthma. His observations contributed to define the biology of asthma as we know it today, and opened the way to the personalised treatment of asthma. His group has successfully continued to investigate the biology and clinical aspects of bronchial asthma, with major interest in the clinical use of biomarkers to monitor disease activity, and in the development of new therapeutic perspectives. This review summarises the latest work on these topics proudly conducted by Maurizio's closest collaborators. The results indicate significant progress in our understanding of asthma in the last 10 years, in particular increased knowledge of the complex interaction between inflammatory and remodelling pathways, improved recognition of biological and clinical asthma phenotypes, and development of new treatment strategies, especially for patients with severe corticosteroid-resistant asthma.

@ERSpublications

Biomarkers may help to define disease phenotypes and new treatment strategies for asthma http://ow.ly/G2Fl6

\section{Introduction}

The life of Maurizio Vignola (fig. 1) was too short, but was sufficient to leave durable signs in the development of a new paradigm for bronchial asthma. Since his first days as a research fellow, first in Montpellier, France, under the guidance of Jean Bousquet, then in Omaha, NE, USA, with Stephen Rennard, he showed innovative thinking and exceptional scientific maturity, a really uncommon finding at his young age. Lifelong friendship and fruitful collaboration were also established in those days with Pascal Chanez, who continued to collaborate with the research group in Palermo, Italy.

Maurizio was very interested in the biology of airway epithelium, and his studies on biopsy specimens from patients with asthma of variable severity allowed him to understand some aspects of the disease that had not been studied in depth. He used immunohistochemistry, a relatively "primitive" technique compared with the sophisticated methods used today, to study several markers of epithelial damage, inflammation, apoptosis, regeneration and remodelling [1-5], which allowed him to propose a new, more complex model of asthma, especially in its most severe form [6]. He addressed the pathogenesis of resistance to corticosteroid treatment [2, 7-9], and also studied the complex relationship between asthma

Received: Nov 172014 | Accepted after revision: Dec 082014

Conflict of interest: None declared.

Provenance: Submitted article, peer reviewed.

Copyright OERS 2015. ERR articles are open access and distributed under the terms of the Creative Commons Attribution Non-Commercial Licence 4.0. 
FIGURE 1 Maurizio Vignola (19642004).

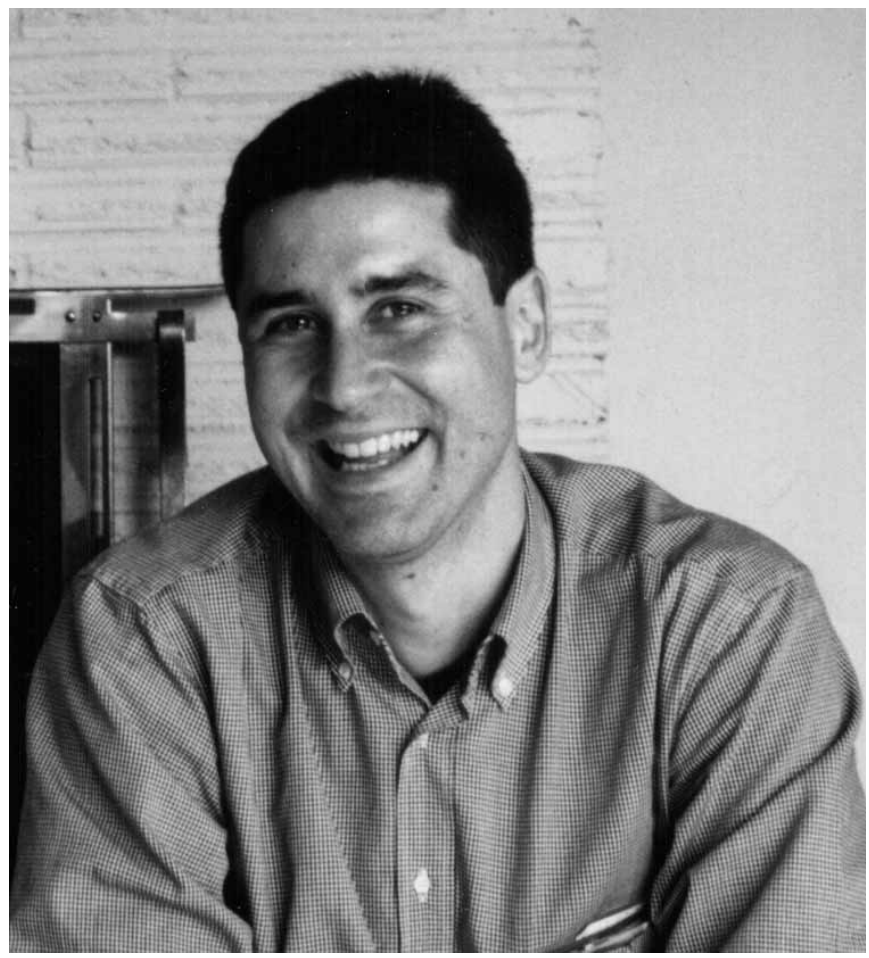

and chronic obstructive pulmonary disease (COPD) when they coexist in the same patient [10]. His research soon extended to extracellular matrix, and he published seminal papers on the role of transforming growth factor (TGF)- $\beta$ [11] and tissue metalloproteinases in asthma [12], setting a new paradigm of the disease that still stands today. He also realised that paediatric asthma could be used to study the earliest aspects of the disease, without the confounding effect of environmental exposures commonly found in adult patients [13]. Finally, he explored the dynamic nature of airway remodelling and the role of myofibroblasts in this process [14].

Space constraints do not allow a thorough discussion of all his scientific papers. Instead, this review focuses on the results obtained in the last few years by his group, who kept working on asthma, first by continuing research on the biology of asthmatic epithelium and on the interactions between upper and lower airway inflammation, and secondly by testing the results obtained through experimental studies in asthmatic patients.

\section{Airway inflammation and remodelling}

Asthma is an inflammatory disorder of the airways, characterised by infiltration of mast cells, eosinophils and T-helper cell type 2 (Th2) $\mathrm{CD}^{+}$T-lymphocytes in the airway wall [15]. Eosinophils within the airways of asthmatics: 1) are recruited via a chemical gradient due to the release of chemotactic agents (interleukin (IL)-5 and eotaxin), 2) show a prolonged survival, and 3) induce epithelial damage and remodelling via the release of eosinophil toxic products (eosinophil peroxidase and the eosinophil cationic protein).

Similarly, the accumulation of T-lymphocytes within the airways of asthmatics may be due to both increased recruitment and decreased cell apoptosis [16]. The increased recruitment of lymphocytes may be promoted by the release of lymphokines including thymic stromal lymphopoietin (TSLP) [17]. TSLP is expressed by structural and immune cells at the site of allergen entry into the airways and has been identified as a master switch for allergic inflammation, since it regulates and drives Th2 responses [18]. Th2 lymphocytes induce and regulatory T-cells (Treg) (characterised by Foxp3 expression) suppress several features of allergic inflammation in asthmatics [15].

The Th2 response, and in particular increased expression of IL-13 within the lungs of asthmatic subjects, triggers an aberrant injury-repair mechanism leading to remodelling of the airway wall. Some of the most prominent effects of IL-13 include increases in goblet cell differentiation, activation of fibroblasts and increases in bronchial responsiveness. Moreover, IL-13 induces bronchial epithelial cells to secrete periostin, which in turn enhances profibrotic TGF- $\beta$, a tissue growth factor whose dysregulation is associated with airway remodelling and might affect disease chronicity and severity [19]. 
Airway epithelium is one of the principal actors in the pathological events associated with both bronchial inflammation and remodelling in asthma [20]. Airway epithelium represents more than a protective barrier against external insults, since it actively secretes pro-inflammatory mediators, i.e. leukotrienes and cytokines such as IL- 8 and TSLP, which in turn amplify the initial inflammation due to external insults by recruiting and activating neutrophils in the early phase, and eosinophils and lymphocytes during the late response. Bronchial epithelial cells are activated by cysteinyl leukotrienes including leukotriene (LT) $\mathrm{D}_{4}$, via activation of extracellular signal-regulated kinase (ERK) $1 / 2$ and of the signal transducer and activator of transcription (STAT)-1, increasing their adhesiveness to eosinophils [21].

Studies on bronchial biopsies demonstrated that in asthmatics, especially if severe, the airway epithelium highly expresses pro-inflammatory transcription factors including nuclear factor (NF)- $\kappa \mathrm{B}[2]$ and cAMP response element binding protein (CREB) [22]. Both transcription factors induce acetylation of chromatin and transcription of pro-inflammatory genes, leading to persistent inflammation and severe disease.

The increased expression of phosphorylated (p)CREB in the airway epithelium is positively correlated with leukocyte infiltration within the bronchi $\left(\mathrm{CD} 45 \mathrm{RB}^{+}\right.$cells), strongly supporting the concept that activation of the airway epithelium is crucial in the recruitment and activation of leukocytes within the airways, as confirmed by the elevated expression of pCREB also in these cells [22]. Furthermore, increased pCREB expression was also observed in peripheral blood mononuclear cells (PBMCs) from untreated and severe asthmatics, suggesting that some of the molecular events crucially involved in the pathogenesis of asthma may be evaluated in systemic compartments, thus avoiding bronchial biopsies to monitor asthma inflammation.

As a further support to the assessment of systemic inflammation to manage asthmatics, it has been shown

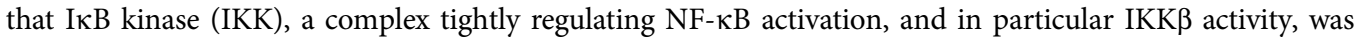
higher in PBMCs in asthmatic patients than in controls [23]. IKK $\beta$ activation seems to be specific for asthma, since increased IKK $\alpha$ activity was found in patients with COPD and healthy smokers compared with asthmatic patients or controls [23]. The different profile in the upstream regulation of the IKK-driven $\mathrm{NF}-\kappa \mathrm{B}$ system in asthmatic and in COPD patients may explain, at least in part, the different pro-inflammatory and pharmacological responses in asthmatic versus COPD patients.

Persistence of chronic inflammation may alter the homeostasis of lung tissue, leading to airway remodelling. Tissue remodelling includes epithelial alterations (epithelial shedding), increased matrix deposition, matrix degradation and accumulation of plasma proteins. Genetic influences, fetal exposures and early life events, and long-term uncontrolled inflammation may all contribute to airway remodelling. Structural changes may alter the protective functions of the airways by affecting mucociliary clearance and increasing the risk of infections and/or exacerbations. Furthermore, activated fibroblasts and smooth muscle cells may release pro-inflammatory mediators, leading to amplification of subsequent tissue damage [24].

The adipokine leptin is a potential new player in bronchial epithelial homeostasis, since it counteracts some molecular events crucially involved in airway remodelling [25]. Leptin decreases the spontaneous release of TGF- $\beta$ and promotes tissue repair by increasing epithelial cell proliferation. Conversely, TGF- $\beta$ decreases leptin receptor expression in the airway epithelium. The bronchial epithelium of asthmatics undergoes shedding, and shows decreased expression of leptin and its receptor, increased thickness of the reticular basement membrane and increased TGF- $\beta$ expression compared with the values found in healthy volunteers [26].

\section{Biomarkers}

Allergic diseases, including allergic rhinitis and asthma, are chronic inflammatory disorders with a prevailing Th2 immune response. Inhalation of allergens leads to bronchial hyperreactivity and recruitment of eosinophils, mast cells and lymphocytes in the upper and lower airways, triggering the inflammatory cascade and generating local and systemic inflammatory responses. Uncontrolled inflammation in the upper and lower airways and in the systemic circulation may compromise the control of allergic rhinitis and asthma, with subsequent disease progression [27]. Therefore, from a clinical point of view, it is important to detect and monitor the occurrence of inflammation and remodelling in the upper and lower airways and in the systemic circulation of patients with asthma and allergic rhinitis. This goal may be achieved using noninvasive methods such as induced sputum, exhaled breath condensate (EBC), nasal wash and collection of blood samples. This approach might also be useful to characterise different inflammatory phenotypes and monitor them in order to provide "personalised" treatment to each individual patient.

Exhaled nitric oxide fraction $(\mathrm{FeNO})$ measurements and collection of $\mathrm{EBC}$ are noninvasive techniques for the assessment of nasal and oral airway inflammation in atopic subjects with and without asthma [28], providing useful clinical information even in the absence of symptoms. In children with atopic dermatitis, allergic rhinitis and asthma, FeNO, and $\mathrm{pH}$ and IL-5 levels in the EBC from upper and lower airways were 
studied to identify children with atopic disorders who might develop bronchial asthma, and to predict the development of exacerbations in asthmatic children [29].

A large number of molecules measured in $\mathrm{EBC}$, including $\mathrm{LTB}_{4}$, prostaglandin $\mathrm{E}_{2}$ and 8-isoprostane, are considered useful markers of airway inflammation and oxidative stress in the airways. In children with persistent rhinitis and asthma of variable severity, such markers and FeNO were measurable in both upper and lower airways, but showed a large variability. Correlations between these markers and their persistence despite treatment might help to identify a relationship between biomarkers in the two airway compartments that might be useful to phenotype the "united allergic disease of the airways" [30].

In asthma, symptoms are often not specific and do not closely reflect the presence or severity of airway inflammation [31]. GAGLIARDO et al. [32] showed that several biomarkers in induced sputum, including IL-8, matrix metalloproteinase (MMP)-9, tissue inhibitor of metalloproteinase (TIMP)-1 and number of eosinophils, as well as the levels of FeNO, were higher in children with intermittent asthma and moderate asthma compared with healthy subjects, suggesting that the inflammatory process is associated with biological and clinical heterogeneity. TGF- $\beta 1$ in induced sputum supernatants was also higher in children with intermittent or moderate asthma compared with controls, and was associated with activation of the intracellular TGF- $\beta /$ Smad signalling pathway in induced sputum cells. Therefore, a dynamic mechanism results from the interaction between TGF- $\beta 1$ signalling activity and inflammatory cells through the commitment of bronchial epithelium, indicating a putative immunoregulatory role of TGF- $\beta 1$ in the progression of airway inflammation in paediatric asthma. As a consequence, treatment strategies should be aimed at controlling inflammation and preventing airway structural changes in young asthmatic patients [33].

Recent studies have identified an IL-23-dependent T-cell population, a Th lineage distinct from Th1 and Th2 called Th17, which plays an important role in inflammation and tissue injury [34]. Th17 ${ }^{+}$cells primarily produce IL-17A, and include $\mathrm{CD} 4^{+}$and $\mathrm{CD}^{+}{ }^{-} \mathrm{T}$-cells showing either the Th1 or Th2 cytokine profile [35]. IL-17 $7^{+}$cells are overexpressed in the lung during acute neutrophilic inflammation and asthma [36]. In mouse models of airway inflammation, such as those driven by house dust mite allergen, ovalbumin [37, 38] or ozone [39], IL-17A controlled bronchial hyperresponsiveness and airway remodelling but did not affect airway eosinophilia and Th2 cell recruitment to the airways [40, 41]. These studies suggest that some of the pathogenic effects of IL-17 are mediated directly on bronchial smooth muscle cells and local fibroblast progenitors, rather than indicating a major effect on airway inflammation. At variance with these experimental results, Albano et al. [42], using noninvasive methods (induced sputum, nasal wash and plasma samples), showed that increased levels of IL-17A are involved in the systemic and upper and lower airway inflammation in children with allergic asthma and rhinitis. In particular, higher levels of IL-17A were documented in nasal wash and induced sputum supernatants of allergic children. In vitro, IL-17A increased the production of IL-8 in nasal and bronchial epithelial cell lines. Moreover, increased levels of intracellular IL-17A and RAR-related orphan receptor (ROR)Ct, associated with lower levels of Foxp3 in T-lymphocytes, together with higher plasma levels of IL-17A, suggest a Th17/Treg imbalance in moderate forms of allergic asthma and rhinitis. Therefore, Th17 immunity appears to be involved in allergic asthma and rhinitis in children, reinforcing the concept of the "united airway disease" in allergic diseases [42]. Despite the progress in our understanding of the pathophysiological functions of Th17 cells in inflammatory diseases of the airways, the regulation of Th17 responses remains relatively poorly understood and controversial. Research on the regulation of Th17 responses in airway disease is quite limited, and the underlying mechanisms have not been thoroughly studied. However, altogether the available studies indicate that the Th17 responses may be differently regulated under in vitro or ex vivo conditions, in animal or human models, in different cell types, or according to variable patterns of endogenous molecules, drugs, steroids or bacterial products in the setting of inflammatory disease [43].

Nebulisers are still frequently used in clinical practice to overcome problems with the technique of inhalers [44], and nebulisation is particularly useful to deliver bronchodilators in children, the elderly and severe or steroid-dependent asthmatics [45]. Profita et al. [46] conducted a randomised, double-blind, placebocontrolled study to determine whether nebulised beclomethasone dipropionate (nBDP) may positively affect inflammation and improve the clinical and functional status in children with both allergic asthma and allergic rhinitis with exclusive exacerbation during the pollen season. nBDP (400 $\mathrm{g}$ twice a day for 4 weeks) was administered through a facial mask, looking at specific markers of inflammation from the nose and bronchi during the season of allergen exposure. Treatment with nBDP reduced the levels of oral FeNO and oral EBC IL-5, while it did not affect the levels of oral $\mathrm{pH}$. In the nasal compartment, nBDP reduced the levels of EBC IL-5 and increased EBC pH, while it did not affect FeNO. Therefore, noninvasive techniques are very useful to study and monitor the effects of treatment, especially in paediatric asthma [46].

Recent studies have also examined the role of vitamin D in allergic diseases. Vitamin D is a potent immune system modulator involved in the development of allergy-associated Th2 cells [47]. BonANNo et al. [48] 
investigated the relationship between different plasma levels of 25-hydroxyvitamin $\mathrm{D}(25(\mathrm{OH}) \mathrm{D})$ in children with allergic disease of the airways. Children with concomitant asthma and rhinitis who showed deficient $25(\mathrm{OH}) \mathrm{D}$ had higher levels of blood eosinophils, a higher number of exacerbations in the last year, a longer disease duration and higher total allergen IgE score and total atopy index compared with children with normal 25(OH)D levels. Therefore, low levels of 25(OH)D in plasma samples could be useful to identify children with allergic rhinitis prone to develop asthma [48].

Stepping forward from the Maurizio Vignola experience, the components of his group are still involved in studies on noninvasive biomarkers of inflammation and remodelling in allergic disease. Together, these studies have contributed to defining the phenotype of disease and to identifying the benefits of a pharmacological approach in the control of the innate and adaptive immunity, and of the related symptoms, in patients with allergic disease of the airways.

\section{Asthma phenotypes and disease control: a new perspective}

In the attempt to better characterise and treat bronchial asthma, several studies have been performed over the last decade to define asthma phenotypes. A variety of parameters have been identified for this definition: functional parameters, frequency of exacerbations, age of patients, presence of allergy, geographical localisation [49] and biomarkers. In a recent pan-European study, the stability of phenotypes in adults with controlled and severe asthma has been analysed in the BIOAIR cohort (longitudinal assessment of clinical course and BIOmarkers in severe chronic AIRway disease study), to compare phenotypes defined either by biomarkers (the "Hastie approach") or by physiological variables (the "Moore approach") over 1 year of observation [50]. There are still conflicting data on the repeatability of inflammatory phenotypes defined by sputum analysis. Fleming et al. [51] found that sputum results were unstable in children with asthma, whereas studies in adults suggested reasonable stability and repeatability of sputum measurements [52, 53]. This BIOAIR study concluded that the inflammatory phenotypes determined by induced sputum cellularity were less stable than those defined by physiological variables, confirming that asthma is a variable inflammatory disease and, therefore, phenotyping to understand mechanisms of the disease needs to be long-term and based on repeated measures, and should ideally integrate several different outcomes [54].

On the basis of these considerations, it is necessary to define what we mean by "asthma control". The most used definition refers to the stability of clinical and functional parameters. A patient with normal spirometry and no symptoms is generally defined as a "controlled" patient. However, some recent papers have shown that the inflammatory profile of an asthmatic patient should also be taken into account in the definition of asthma control. In this regard, within populations of patients with allergic rhinitis, intermittent asthma (treated only with short-acting $\beta_{2}$-agonists (SABA) on demand) or moderate asthma (treated with inhaled corticosteroids (ICS) and long-acting $\beta_{2}$-agonists plus SABA on demand), and also within a population of patients with atopic dermatitis, some subjects show ongoing bronchial inflammation, i.e. low pH and high IL-5 concentrations in the EBC, as well as increased FeNO levels [29]. Therefore, some specific "phenotypes" of these patient populations show evidence of bronchial inflammation. Although these patients were not treated with ICS because they did not have bronchial asthma (i.e. atopic dermatitis or rhinitis) or had intermittent asthma, they were probably representing a specific phenotype of patients who may develop symptoms on an accelerated "allergic march". Moreover, within the group of patients with moderate asthma, who were treated with ICS, some of them displayed bronchial inflammation according to the above-mentioned biomarkers, suggesting that these patients might have been candidate to "step up" in the classification of their disease [29]. Accordingly, within a population of patients with intermittent asthma, some subjects displayed increased expression of pCREB and its receptor expression [22], and low expression of leptin and its receptor [26] in bronchial epithelium, suggesting that in these patients bronchial inflammation occurs and can be demonstrated at the tissue level. These latter studies also demonstrate that bronchial tissue inflammation occurs in some patients with moderate asthma treated with ICS, suggesting that "specific phenotypes" of not fully controlled inflammation do occur in patients.

Partially or not controlled disease, as indicated by positive biomarkers of inflammation in asthma, may have important consequences on treatment. The longitudinal study conducted in paediatric patients with asthma discussed earlier [32] confirmed that a specific phenotype of controlled disease on the basis of symptoms and spirometry, with increased bronchial inflammation on the basis of inflammatory biomarkers, is associated with poorly controlled disease and frequent exacerbations.

As already mentioned, the involvement of the upper airways strongly influences the control of bronchial asthma both from a functional and an inflammatory point of view. In a pan-European study conducted by the Global Allergy and Asthma European Network (GA $\left.{ }^{2} L E N\right)$, chronic rhinosinusitis was positively associated with adult-onset asthma. This association has been interpreted as chronic rhinosinusitis causing 
asthma, because of the release of inflammatory mediators and cytokines from the diseased sinus tissue into the circulation and recruitment of eosinophils into both upper and lower airways from the bone marrow and circulation [55]. These observations confirm that patients with asthma and chronic rhinosinusitis represent a phenotype where upper airway inflammation has to be controlled to achieve a complete control of asthma. Chronic rhinosinusitis and asthma are linked by a common inflammatory pathway, in which eosinophils and airway epithelium play an important role [56, 57]. Many asthmatic patients, whatever their severity, have chronic rhinosinusitis demonstrated on computed tomography scans. Patients with severe asthma, uncontrolled asthma or both have more severe computed tomography scan abnormalities than other patients. In many patients with severe asthma, surgical treatment of chronic rhinosinusitis or nasal polyposis might improve asthma symptoms [57]. Accordingly, a recent study from our group used an asthma-specific gene array to assess the inflammatory profile of nasal biopsies from patients with chronic rhinosinusitis and nasal polyps [58]. Among the cytokines whose expression was altered, IL-19, a cytokine contributing to the increased activation of Th2 lymphocytes, was increased in patients with chronic rhinosinusitis with nasal polyps [58]. Moreover, IL-19 protein was found to be highly expressed in the metaplastic nasal epithelium when compared with normal or hyperplastic epithelium [58]. IL-19 could therefore contribute to the increased proliferation of nasal epithelial cells and to remodelling processes in these patients.

Phenotyping patients with bronchial asthma represents an important strategy to achieve control of this complex disease. Future therapeutic strategies will certainly take into account the characterisation of these "phenotypes" made on the basis of the predominant pathogenetic pathway(s) of inflammatory processes.

\section{Mechanisms in glucocorticoid control of inflammation}

Glucocorticosteroids are widely used to treat various inflammatory and immune diseases. The most common use of glucocorticosteroids today is in the treatment of asthma and other allergic diseases, and inhaled glucocorticosteroids have now become established as a first-line treatment in adults and children who have persistent asthma. Suppression of inflammation by corticosteroids positively affects asthma symptoms and airway inflammation in most patients. The major action of glucocorticosteroids is to switch off activated inflammatory genes that encode for cytokines, chemokines, adhesion molecule, inflammatory enzymes and receptors [59].

Several studies have examined the efficacy of glucocorticosteroid-based therapies in airway inflammation, cell activation and immunological responses. PACE et al. [60] showed that treatment with budesonide was effective in controlling asthma inflammation by reducing both T-lymphocyte activation and survival and inducible T-cell co-stimulator (ICOS) in $\mathrm{CD}^{+} / \mathrm{CD} 25^{-}$cells, and by increasing survival and activation of $\mathrm{CD}^{+} / \mathrm{CD} 25^{+} \mathrm{Foxp}^{+}$cells (Treg) both in vitro and in vivo. Furthermore, fluticasone propionate was shown to control peripheral blood T-cell activation and induce T-cell apoptosis through activation of caspases 8 and 3 in mild-persistent asthmatics [61]. Vignola et al. [62] showed that in mild-persistent asthmatics, low-dose fluticasone propionate was effective in improving symptoms due, at least in part, to the resolution of eosinophilic bronchial inflammation and downregulation of remodelling markers, such as MMP-9 and TIMP-1 levels in induced sputum samples. Other in vitro studies confirmed these findings: flunisolide treatment of sputum cells isolated from asthmatic subjects with mild-to-moderate asthma inhibited the release of MMP-9, TIMP-1, TGF- $\beta$ and fibronectin and increased eosinophil apoptosis [63]. Exposure of induced sputum cells from mild to moderate asthmatic subjects to beclomethasone dipropionate reduced granulocyte-macrophage colony-stimulating factor, RANTES and IL-8 levels and induced transcription of the $\beta_{2}$ receptor [64]. Furthermore fluticasone furoate is able to upregulate the leptin/leptin receptor pathway also in nasal epithelial cells, thus increasing epithelial homeostasis upon allergen exposure [65].

Understanding molecular mechanisms whereby corticosteroids suppress inflammation explains how glucocorticosteroids are able to inhibit inflammatory pathways, and also provides insights into the complex molecular mechanisms of steroid unresponsiveness. glucocorticosteroids activate and suppress many proand anti-inflammatory genes, such as NF- $\kappa \mathrm{B}$ and correlated kinase proteins [59]. Several distinct molecular mechanisms contributing to decreased anti-inflammatory effects of glucocorticosteroids have been identified. Excessive activation of NF- $\kappa B$ can cause reduced or absent responsiveness to glucocorticoids in asthma, since NF- $\mathrm{KB}$ binds the glucocorticoid receptor, preventing its interaction with the glucocorticoid response element and other transcription factors. PBMCs from uncontrolled severe adult asthmatics showed higher levels of NF- $\kappa \mathrm{B}$ p65 protein expression, $\mathrm{I} \kappa \mathrm{B} \alpha$ phosphorylation and IKK $\beta$ kinase activity than normal individuals, despite long-term inhaled and oral glucocorticosteroid treatment $[9,23]$. A similar state of overexpression and activation of NF- $\mathrm{B}$ p65 subunit protein in PBMCs and induced sputum cells has been shown in children with moderate to severe asthma [13, 32].

Activated glucocorticoid receptor interacts with co-repressor molecules to attenuate NF- $\kappa \mathrm{B}$-associated co-activator activity, thus reducing histone acetylation and chromatin remodelling. Chromatin modification 
plays a critical role in the regulation of inflammatory genes and there is an extensive literature concerning histone acetylation and mechanism of action of glucocorticosteroids. CHIAPPARA et al. [22] showed that the increased expression of pCREB can be modulated by glucocorticoids in responsive asthmatic patients.

Stepping forward from the Maurizio Vignola experience, our research group has further contributed to the definition of the molecular mechanisms in glucocorticoid control of inflammation. The new insights into corticosteroid action may lead to new pharmacological approaches to treat inflammatory lung diseases, and in particular to counteraction of reduced steroid responsiveness in complex inflammatory diseases, such as asthma.

\section{New therapies in corticosteroid-resistant asthma}

Since multiple asthma phenotypes with distinct pathogenetic profiles have been identified, a personalised strategy in treatment directed towards disease-specific features is expected to improve outcomes in these specific patients [66].

Allergic asthma is characterised by increased levels of IgE. Omalizumab, a humanised anti-IgE monoclonal antibody specifically binding free IgE, has been introduced in asthma treatment. Omalizumab interrupts the allergic cascade by preventing the binding of IgE with its high-affinity FceRI receptors on target cells (mast cells, basophils, antigen-presenting cells, etc.) [67]. Its clinical efficacy has been well documented in a number of clinical trials in patients with moderate-to-severe and severe allergic asthma and with increased levels of IgE. Omalizumab reduced exacerbations, asthma symptoms and use of ICS and rescue medication, and improved quality of life relative to placebo [68]. Results from recent pooled data from randomised clinical trials and from a large prospective cohort study provided reassurance about the long-term safety of omalizumab. Long-term treatment with omalizumab was able to reduce inflammation [69] and improved clinical control in severe asthmatics [70]. In severe uncontrolled asthma, omalizumab increased Foxp3 expression and decreased the pCREB/CREB ratio and IL-8 expression. These findings correlated with the forced expiratory volume in $1 \mathrm{~s}$ (FEV1) values and symptom scores [70].

Another asthma phenotype is characterised by increased numbers of neutrophils in the airways of a subgroup of patients with severe asthma, characterised by inadequate symptom control and high rate of inflammation despite high doses of steroids. Since IL-8, a neutrophil chemotactic agent, exerts pro-inflammatory responses in leukocytes by activating the G-protein coupled receptor CXCR2, a selective CXCR2 receptor antagonist (SCH 527123) was tested in a randomised clinical study in patients with severe asthma and increased number of neutrophils in sputum. A mean reduction of $36.3 \%$ in sputum neutrophil percentage was shown in the treated arm, compared with a $6.7 \%$ increase in the placebo arm. In addition, asthmatics treated with SCH 527123 experienced fewer mild exacerbations without additional adverse events compared with the placebo group [71]. Conversely, a randomised clinical study on the use of golimumab $(50,100$ or $200 \mathrm{mg}$ ), a human monoclonal antibody against tumour necrosis factor (TNF)- $\alpha$, did not demonstrate a favourable risk-benefit profile in patients with severe persistent asthma [72]. Golimumab-treated asthmatics experienced major adverse events, including serious infections, without any advantage in terms of asthma control or improved FEV1. The potential use of anti-TNF monoclonal antibodies in asthmatics remains undefined, since this study showed inaccurate selection of severe asthmatics, i.e. patients were not selected based on elevated levels of TNF before treatment.

To further underline the need for accurate phenotyping of asthmatic patients, a recent clinical trial using the anti-IL-13 lebrikizumab demonstrated that patients with high pre-treatment levels of serum periostin had greater improvement in lung function with lebrikizumab than did patients with low periostin levels [73].

New advanced biotechnologies may provide further perspectives in the therapy of severe asthma by re-directing the immune and inflammatory responses in these patients. In this regard, synthetic hypoallergenic fragments of Parietaria judaica upregulated innate immunity receptors and increased interferon- $\gamma$, and appeared capable of re-orienting the immune system towards a more physiological balance between Th1 and Th2 responses [74]. In summary, personalised therapy aimed at targeting disease-specific features will allow better control in asthmatics, but further studies are still needed to achieve this important goal.

Finally, nonpharmacological therapeutic approaches could also be useful in asthma treatment. Physical exercise has been shown, in animal models of allergic asthma, to significantly reduce airway inflammation [75]. In healthy humans, studies on induced sputum cells at rest and after a marathon [76] or half-marathon [77] race documented that intense exercise caused mild epithelial injury and influx of neutrophils into the airways, possibly due to hyperosmolarity of the airway lining fluid secondary to the hyperpnoea of exercise. However, no significant activation of inflammatory cells occurred [76, 77], in line with the anti-inflammatory effects of exercise documented in animal models. However, such inflammation could negatively affect airway muscle tone. Subsequent studies in healthy runners showed that trained 
subjects were less responsive to single-dose methacholine inhaled in the absence of deep breaths [78], suggesting a positive effect of repeated airway smooth muscle stretching during exercise hyperpnoea. Moreover, healthy sedentary subjects showed decreased bronchial responsiveness after 12 weeks of training [79], and the same effect occurred in sedentary subjects with mild asthma undergoing the same training protocol [80]. Similar results of decreased bronchial responsiveness have been recently reported after application of continuous positive airway pressure (CPAP) during sleep for a week in asthmatic patients free from sleep disordered breathing [81]. Population studies also documented an inverse relationship between physical activity and bronchial responsiveness [82]. Therefore, exercise training might be beneficial in asthmatics, and mechanical stretch of the airways is a possible mechanism of the beneficial effects of exercise or CPAP. However, more data are needed before these results can be applied clinically.

\section{Conclusion}

Maurizio Vignola enthusiastically set up a solid research group that in the last 10 years has carried forward the large amount of work that he had so successfully started. Research activity has continued on both the experimental and the clinical aspects of asthma, with special regard to the biology of inflammation and remodelling of the airways, the relationship between upper and lower airway inflammation, and the complex mechanisms responsible for steroid-resistant asthma. On the clinical side, the group is part of European networks and actively contributes to the studies on the definition of asthma phenotypes based on biomarkers. Finally, studies on new treatment strategies will help to achieve the personalised treatment of asthma that Maurizio Vignola had foreseen as "the future" for asthma patients.

\section{Acknowledgements}

More people than those directly contributing to this review article collaborated with Maurizio Vignola on asthma projects: Anna Bonanno, Andreina Bruno, Maria Ferraro, Stefania La Grutta, Mario Melis, Anna Merendino, Liboria Siena, Fabio Bucchieri, Laura Chimenti, Giorgia Costanzo, Rossana Di Giorgi, Francesca Guddo, Daniela Guerrera, Franco Mirabella, Alessandra Paternò, Aroldo Rizzo, Nicola Scichilone and Annalisa Tipa. Many of them still work at the CNR Institute of Biomedicine and Molecular Immunology or at the University of Palermo (both Palermo, Italy), and their contribution is acknowledged. Finally, a special acknowledgment is dedicated to Giovanni Bonsignore, Professor Emeritus of Respiratory Medicine (1927-2007), who introduced many young fellows, including Maurizio Vignola, to respiratory basic and clinical research.

\section{References}

1 Vignola AM, Chanez P, Campbell AM, et al. Airway inflammation in mild intermittent and in persistent asthma. Am J Respir Crit Care Med 1998; 157: 403-409.

2 Vignola AM, Chiappara G, Siena L, et al. Proliferation and activation of bronchial epithelial cells in corticosteroid-dependent asthma. J Allergy Clin Immunol 2001; 108: 738-746.

3 Vignola AM, Bonsignore G, Siena L, et al. ICAM-1 and $\alpha 3 \beta 1$ expression by bronchial epithelial cells and their in vitro modulation by inflammatory and anti-inflammatory mediators. Allergy 2000; 55: 931-939.

4 Merendino AM, Paul C, Vignola AM, et al. Heat shock protein-27 protects human bronchial epithelial cells against oxidative stress-mediated apoptosis: possible implication in asthma. Cell Stress Chaperones 2002; 7: 269-280.

5 Vignola AM, Chanez P, Chiappara G, et al. Evaluation of apoptosis of eosinophils, macrophages, and T lymphocytes in mucosal biopsy specimens of patients with asthma and chronic bronchitis. J Allergy Clin Immunol 1999; 103: 563-573.

6 Bousquet J, Jeffery PK, Busse WW, et al. Asthma. From bronchoconstriction to airways inflammation and remodeling. Am J Respir Crit Care Med 2000; 161: 1720-1745.

7 Vachier I, Chiappara G, Vignola AM, et al. Glucocorticoid receptors in bronchial epithelial cells in asthma. Am J Respir Crit Care Med 1998; 158: 963-970.

8 Gagliardo R, Chanez P, Vignola AM, et al. Glucocorticoid receptor $\alpha$ and $\beta$ in glucocorticoid dependent asthma. Am J Respir Crit Care Med 2000; 162: 7-13.

9 Gagliardo R, Chanez P, Mathieu M, et al. Persistent activation of nuclear factor- $\mathrm{\kappa B}$ signaling pathway in severe uncontrolled asthma. Am J Respir Crit Care Med 2003; 168: 1190-1198.

10 Chanez P, Vignola AM, O'Shaugnessy $\mathrm{T}$, et al. Corticosteroid reversibility in COPD is related to features of asthma. Am J Respir Crit Care Med 1997; 155: 1529-1534.

11 Vignola AM, Chanez P, Chiappara G, et al. Transforming growth factor- $\beta$ expression in mucosal biopsies in asthma and chronic bronchitis. Am J Respir Crit Care Med 1997; 156: 591-599.

12 Vignola AM, Riccobono L, Mirabella A, et al. Sputum metalloproteinase-9/tissue inhibitor of metalloproteinase-1 ratio correlates with airflow obstruction in asthma and chronic bronchitis. Am J Respir Crit Care Med 1998; 158: $1945-1950$.

13 La Grutta S, Gagliardo R, Mirabella F, et al. Clinical and biological heterogeneity in children with moderate asthma. Am J Respir Crit Care Med 2003; 167: 1490-1495.

14 Vignola AM, Kips J, Bousquet J. Tissue remodeling as a feature of persistent asthma. J Allergy Clin Immunol 2000; 105: 1041-1053.

15 Lambrecht BN, Hammad H. Asthma: the importance of dysregulated barrier immunity. Eur J Immunol 2013; 43: 3125-3137.

16 Melis M, Siena L, Pace E, et al. Fluticasone induces apoptosis in peripheral T-lymphocytes: a comparison between asthmatic and normal subjects. Eur Respir J 2002; 19: 257-266.

17 Zhou B, Comeau MR, De Smedt T, et al. Thymic stromal lymphopoietin as a key initiator of allergic airway inflammation in mice. Nat Immunol 2005; 6: 1047-1053. 

Ther 2008; 325: 1024-1030.

22 Chiappara G, Chanez P, Bruno A, et al. Variable p-CREB expression depicts different asthma phenotypes. Allergy 2007; 62: 787-794.

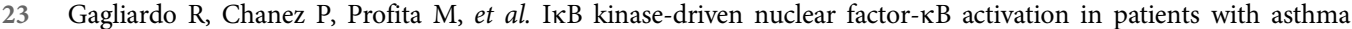
and chronic obstructive pulmonary disease. J Allergy Clin Immunol 2011; 128: 635-645.

24 Bousquet J, Jacot W, Yssel H, et al. Epigenetic inheritance of fetal genes in allergic asthma. Allergy 2004; 59: $138-147$.

25 Vernooy JH, Ubags ND, Brusselle GG, et al. Leptin as regulator of pulmonary immune responses: involvement in respiratory diseases. Pulm Pharmacol Ther 2013; 26: 464-472.

26 Bruno A, Pace E, Chanez P, et al. Leptin and leptin receptor expression in asthma. J Allergy Clin Immunol 2009; 124: 230-237.

27 Bourdin A, Gras D, Vachier I, et al. Upper airway. 1: Allergic rhinitis and asthma: united disease through epithelial cells. Thorax 2009; 64: 999-1004.

28 Griese M, Latzin P, Beck J. A noninvasive method to collect nasally exhaled air condensate in humans of all ages. Eur J Clin Invest 2001; 31: 915-920.

29 Profita M, La Grutta S, Carpagnano E, et al. Noninvasive methods for the detection of upper and lower airway inflammation in atopic children. J Allergy Clin Immunol 2006; 118: 1068-1074.

30 Profita M, Montuschi P, Bonanno A, et al. Novel perspectives in the detection of oral and nasal oxidative stress and inflammation in pediatric united airway diseases. Int J Immunopathol Pharmacol 2010; 23: 1211-1219.

31 van den Toorn LM, Overbeek SE, de Jongste JC, et al. Airway inflammation is present during clinical remission of atopic asthma. Am J Respir Crit Care Med 2001; 164: 2107-2113.

32 Gagliardo R, La Grutta S, Chanez P, et al. Non-invasive markers of airway inflammation and remodeling in childhood asthma. Pediatr Allergy Immunol 2009; 20: 780-790.

33 Gagliardo R, Chanez P, Gjomarkaj M, et al. The role of transforming growth factor- $\beta 1$ in airway inflammation of childhood asthma. Int J Immunopathol Pharmacol 2013; 26: 725-738.

34 Oboki K, Ohno T, Saito H, et al. Th17 and allergy. Allergol Int 2008; 57: 121-134.

35 Robinson DS. Regulatory T cells and asthma. Clin Exp Allergy 2009; 39: 1314-1323.

36 Alcorn JF, Crowe CR, Kolls JK. TH17 cells in asthma and COPD. Annu Rev Physiol 2010; 72: $495-516$.

37 Kudo M, Melton AC, Chen C, et al. IL-17A produced by $\alpha \beta \mathrm{T}$ cells drives airway hyper-responsiveness in mice and enhances mouse and human airway smooth muscle contraction. Nat Med 2012; 18: 547-554.

38 Wang Q, Li H, Yao Y, et al. The overexpression of heparin-binding epidermal growth factor is responsible for Th17-induced airway remodeling in an experimental asthma model. J Immunol 2010; 185: 834-841.

39 Pichavant M, Goya S, Meyer EH, et al. Ozone exposure in a mouse model induces airway hyperreactivity that requires the presence of natural killer T cells and IL-17. J Exp Med 2008; 205: 385-393.

40 Bellini A, Marini MA, Bianchetti L, et al. Interleukin (IL)-4, IL-13, and IL-17A differentially affect the profibrotic and proinflammatory functions of fibrocytes from asthmatic patients. Mucosal Immunol 2012; 5: 140-149.

41 Zhao J, Lloyd CM, Noble A. Th17 responses in chronic allergic airway inflammation abrogate regulatory T-cell-mediated tolerance and contribute to airway remodeling. Mucosal Immunol 2013; 6: 335-346.

42 Albano GD, Di Sano C, Bonanno A, et al. Th17 immunity in children with allergic asthma and rhinitis: a pharmacological approach. PLoS One 2013; 8: e58892.

43 Liu Y, Zeng M, Liu Z. Th17 response and its regulation in inflammatory upper airway diseases. Clin Exp Allergy 2014 [In press DOI: 10.1111/cea.12378].

44 Szefler SJ, Baker JW, Uryniak T, et al. Comparative study of budesonide inhalation suspension and montelukast in young children with mild persistent asthma. J Allergy Clin Immunol 2007; 120: 1043-1050.

45 Bousquet J, Delacourt C. Conclusion. Nebulized beclometasone dipropionate therapy in asthma. Respir Med 2003; 97: Suppl B, S41.

46 Profita M, Riccobono L, Bonanno A, et al. Effect of nebulized beclomethasone on airway inflammation and clinical status of children with allergic asthma and rhinitis: a randomized, double-blind, placebo-controlled study. Int Arch Allergy Immunol 2013; 161: 53-64.

47 Benson AA, Toh JA, Vernon N, et al. The role of vitamin D in the immunopathogenesis of allergic skin diseases. Allergy 2012; 67: 296-301.

48 Bonanno A, Gangemi S, La Grutta S, et al. 25-Hydroxyvitamin D, IL-31, and IL-33 in children with allergic disease of the airways. Mediators Inflamm 2014; 2014: 520241.

49 Newson RB, van Ree R, Forsberg B, et al. Geographical variation in the prevalence of sensitization to common aeroallergens in adults: the GA²LEN survey. Allergy 2014; 69: 643-651.

50 Kupczyk M, ten Brinke A, Sterk PJ, et al. Frequent exacerbators - a distinct phenotype of severe asthma. Clin Exp Allergy 2014; 44: 212-221.

51 Fleming L, Tsartsali L, Wilson N, et al. Sputum inflammatory phenotypes are not stable in children with asthma. Thorax 2012; 67: 675-681.

52 Pizzichini E, Pizzichini MM, Efthimiadis A, et al. Indices of airway inflammation in induced sputum: reproducibility and validity of cell and fluid-phase measurements. Am J Respir Crit Care Med 1996; 154: 308-317.

53 McGrath KW, Icitovic N, Boushey HA, et al. A large subgroup of mild-to-moderate asthma is persistently noneosinophilic. Am J Respir Crit Care Med 2012; 185: 612-619.

54 Jarvis D, Newson R, Lotvall J, et al. Asthma in adults and its association with chronic rhinosinusitis: the GA ${ }^{2} \mathrm{LEN}$ survey in Europe. Allergy 2012; 67: 91-98.

55 Bousquet J, Jacot W, Vignola AM, et al. Allergic rhinitis: a disease remodeling the upper airways? J Allergy Clin Immunol 2004; 113: 43-49. 
56 Watelet JB, Van Zele T, Gjomarkaj M, et al. Tissue remodelling in upper airways: where is the link with lower airway remodelling? Allergy 2006; 61: 1249-1258.

57 Bousquet J, Bachert C, Canonica GW, et al. Unmet needs in severe chronic upper airway disease (SCUAD). J Allergy Clin Immunol 2009; 124: 428-433.

58 Pace E, Scafidi V, Di Bona D, et al. Increased expression of IL-19 in the epithelium of patients with chronic rhinosinusitis and nasal polyps. Allergy 2012; 67: 878-886.

59 Barnes PJ. Biochemical basis of asthma therapy. J Biol Chem 2011; 286: 32899-32905.

60 Pace E, Di Sano C, La Grutta S, et al. Multiple in vitro and in vivo regulatory effects of budesonide in $\mathrm{CD}_{4}^{+}$ T lymphocyte subpopulations of allergic asthmatics. PLoS One 2012; 7: e48816.

61 Pace E, Gagliardo R, Melis M, et al. Synergistic effects of fluticasone propionate and salmeterol on in vitro T-cell activation and apoptosis in asthma. J Allergy Clin Immunol 2004; 114: 1216-1223.

62 Vignola AM, Riccobono L, Profita $\mathrm{M}$, et al. Effects of low doses of inhaled fluticasone propionate on inflammation and remodelling in persistent-mild asthma. Allergy 2005; 60: 1511-1517.

63 Profita M, Gagliardo R, Di Giorgi R, et al. In vitro effects of flunisolide on MMP-9, TIMP-1, fibronectin, TGF- $\beta 1$ release and apoptosis in sputum cells freshly isolated from mild to moderate asthmatics. Allergy 2004; 59: 927-932.

64 Profita M, Gagliardo R, Di Giorgi R, et al. Biochemical interaction between effects of beclomethasone dipropionate and salbutamol or formoterol in sputum cells from mild to moderate asthmatics. Allergy 2005; 60: 323-329.

65 Bruno A, Gerbino S, Ferraro M, et al. Fluticasone furoate maintains epithelial homeostasis via leptin/leptin receptor pathway in nasal cells. Mol Cell Biochem 2014; 396: 55-65.

66 Chung KF. New treatments for severe treatment-resistant asthma: targeting the right patient. Lancet Respir Med 2013; 1: 639-652.

67 Holgate ST. New strategies with anti-IgE in allergic diseases. World Allergy Organ J 2014; 7: 17.

68 Normansell R, Walker S, Milan SJ, et al. Omalizumab for asthma in adults and children. Cochrane Database Syst Rev 2014; 1: CD003559.

69 Pace E, Ferraro M, Bruno A, et al. Anti-inflammatory effects of seven years treatment with omalizumab in severe uncontrolled asthmatics. Allergy 2010; 65: 1495-1496.

70 Pace E, Ferraro M, Bruno A, et al. Clinical benefits of 7 years of treatment with omalizumab in severe uncontrolled asthmatics. J Asthma 2011; 48: 387-392.

71 Nair P, Gaga M, Zervas E, et al. Safety and efficacy of a CXCR2 antagonist in patients with severe asthma and sputum neutrophils: a randomized, placebo-controlled clinical trial. Clin Exp Allergy 2012; 42: 1097-1103.

72 Wenzel SE, Barnes PJ, Bleecker ER, et al. A randomized, double-blind, placebo-controlled study of tumor necrosis factor- $\alpha$ blockade in severe persistent asthma. Am J Respir Crit Care Med 2009; 179: 549-558.

73 Corren J, Lemanske RF, Hanania NA, et al. Lebrikizumab treatment in adults with asthma. N Engl J Med 2011; 365: 1088-1098.

74 Pace E, Duro G, La Grutta S, et al. Hypoallergenic fragment of Par j 2 increases functional expression of Toll-like receptors in atopic children. Allergy 2006; 61: 1459-1466.

75 Pastva A, Estell K, Schoeb TR, et al. Aerobic exercise attenuates airway inflammatory responses in a mouse model of atopic asthma. J Immunol 2004; 172: 4520-4526.

76 Bonsignore MR, Morici G, Riccobono L, et al. Airway inflammation in nonasthmatic amateur runners. Am J Physiol Lung Cell Mol Physiol 2001; 281: L668-L676.

77 Chimenti L, Morici G, Paternò A, et al. Bronchial epithelial damage after a half-marathon in nonasthmatic amateur runners. Am J Physiol Lung Cell Mol Physiol 2010; 298: L857-L862.

78 Scichilone N, Morici G, Marchese R, et al. Reduced airway responsiveness in nonelite runners. Med Sci Sports Exerc 2005; 37: 2019-2025.

79 Scichilone N, Morici G, Zangla D, et al. Effects of exercise training on airway responsiveness and airway cells in healthy subjects. J Appl Physiol 2010; 109: 288-294.

80 Scichilone N, Morici G, Zangla D, et al. Effects of exercise training on airway closure in asthmatics. J Appl Physiol 2012; 113: 714-718.

81 Busk M, Busk N, Puntenney P, et al. Use of continuous positive airway pressure reduces airway reactivity in adults with asthma. Eur Respir J 2013; 41: 317-322.

82 Shaaban R, Leynaert B, Soussan D, et al. Physical activity and bronchial hyperresponsiveness: European Community Respiratory Health Survey II. Thorax 2007; 62: 403-410. 